\section{The impact of COVID-19 first wave on long term care facilities of an Italian Province: an historical reference}

\author{
Andrea Tramarin, ${ }^{1}$ \\ Nicola Gennaro, ${ }^{2}$ \\ Giancarlo Dal Grande, ${ }^{3}$ \\ Luciana Bragagnolo, ${ }^{4}$ \\ Maria Rosa Carta, ${ }^{5}$ \\ Davide Giavarina, ${ }^{5}$ \\ Michela Pascarella, ${ }^{4}$ \\ Mario Rassu, ${ }^{4}$ \\ Antonio Matteazzi, ${ }^{1}$ \\ Giampaolo Stopazzolo \\ ${ }^{1}$ Primary Health Care, AULSS8, \\ Vicenza; ${ }^{2}$ Epidemiological service of the \\ Veneto Region, Padua; ${ }^{3}$ Don Bruzzo \\ Long Term Care Facility, Gambellara \\ (VI); ${ }^{4}$ Laboratory of Microbiology of \\ San Bortolo Hospital, AULSS8 (VI); \\ ${ }^{5}$ Laboratory of Clinical Chemistry of \\ San Bortolo Hospital, AULSS8 (VI), \\ Italy
}

\section{Abstract \\ The coronavirus disease 2019} (COVID-19) pandemic will leave a profound imprint in the collective memory of humanity. In Italy, Long-Term Care Facilities (LTCFs) have seen a disproportionally high number of deaths during and the COVID-19 pandemic and, certainly, they may be considered as its epicenter. Aiming to leave a symbolic mark of what the pandemic did in these care settings, we report on an outbreak in a single LTCF where, 53 out of 64 residents, resulted infected. Our narration is based on an epidemiological field investigation together with a calendar of passages through the stages of disease in the infected population. We found an age-gradient in all clinical and epidemiological variables explored such as symptoms onset, illness severity, recovery from symptoms and deaths. According to the disease staging, 26 (49\%) were asymptomatic; 9 (17\%) had a mild disease; $7(13 \%)$ a moderate stage and 11 $(21 \%)$ a severe illness severity of whom 10 died. For a more comprehensive description of the impact of the pandemic on LTCFs, we compared the standard mortality ratio (SMR) in the first six months of 2020 to that of 2018 and 2019 in all the 34 facilities of the Vicenza province. Overall, there was a SMR higher $60 \%$ than the equivalent period of the previous years.

\section{Introduction}

On December 12, 2019, 27 cases of pneumonia of unknown causes were reported in Wuhan, Hubei Province, China. With the evolving pandemic, the coronavirus disease 2019 (COVID-19) spread rapidly from China around the entire world. Italy was the first European nation to be affected by COVID-19. On January 31, 2020, two Chinese tourists resulted positive at the severe acute respiratory syndrome-related coronavirus 2 (SARS-CoV-2) swab test in Rome. An outbreak of 16 confirmed cases, never been travelling from and to China, were then reported in the Veneto and in the Lombardy Regions.

The number of cases rose rapidly with a geometric progression in the two Regions (Veneto and Lombardy) and through all the country. In response to the growing pandemic of COVID-19, the Italian government imposed a national quarantine, restricting the movement of the population except for necessities such as work and health circumstances. On May 31, the Italian Ministry of Health reported 233,515 confirmed total cases and 33,530 deaths. $^{2}$

Preliminary studies found that, at a community-level, COVID-19 had a rapid spread and high morbidity and mortality among older adults in Long Term Care Facilities (LTCFs) A retrospective analysis of individual cases data from China, and elsewhere, showed a strong age gradient in the case fatality ratio. ${ }^{3-5}$ Cardiovascular diseases, hypertension, diabetes mellitus were the comorbidities most frequently associated with COVID-19. All these comorbidities are common among dependent elderly housed in institution and many elderly died by the association between their original comorbidities and the novel virus. ${ }^{6}$

In the initial emergency, data from LTCFs not only stressed the vulnerability of their patients and residents and this led to national headlines. Major Italian newspapers reported figures and accounts of incredibly high numbers of deaths in residential care settings denouncing lack of guidelines, medical procedures, testing for COVID-19 and supply of personal protective equipment (PPE). The National Institute of Health has done a dedicated survey in the month of April 2020. The responding LTCFs reported a mortality of $8.4 \%$ in the month of March. Among the 3859 total deaths, only 133 were officially classified as COVID-19 after appropriate testing though, 1310 had flu and COVID19 related symptoms. ${ }^{7}$ The National Institute of Health affirmed that these two
Correspondence: Andrea Tramarin, Primary

Health Care, AULSS8, Contrà Lodi 48,

36100 Vicenza, Italy.

E-mail: tramarinandrea@gmail.com

Key words: COVID-19; aging; mortality;

Long Term Care Facilities.

Contributions: all authors listed in the front page gave substantial contributions to the conception, design of the paper, acquisition and analysis of data. All of them approved the final version of the paper for its publication.

Conflict of interests: the authors declare no potential conflict of interests.

Availability of data and materials: The data used to support the findings of this study are available from the corresponding author upon request.

Ethical approval: this study was approved by the Ethical Committee of Azienda Socio Sanitaria ULSS 8 (Del.694 del 27.05.20).

Preprint: a preprint of the paper has been published by MedRxiv (doi: https://doi.org/ 10.1101/2020.10.21.20216705)

Received for publication: 1 February 2021.

Revision received: 9 March 2021

Accepted for publication: 10 March 2021.

This work is licensed under a Creative Commons Attribution-NonCommercial 4.0 International License (CC BY-NC 4.0).

${ }^{\circ}$ Copyright: the Author(s), 2021

Licensee PAGEPress, Italy

Geriatric Care 2021; 7:9654

doi:10.4081/gc.2021.9654

numbers should be analyzed jointly accounting for the $37.4 \%$ of the deaths of the period as COVID-19 related. ${ }^{6}$ However, the real impact in terms of mortality in LTCFs by COVID-19 is still unknown in Italy.

This paper describes the impact of COVID-19 in the LTCFs of the Vicenza Province (Italy). It is structured into two parts: first, it focuses on an outbreak in a single LTCF where over eighty per cent of an LTCF residents resulted positives to COVID-19. The distribution of COVID-19 clinical manifestations and the risk factors associated to different health outcomes have been analyzed. Secondly, an epidemiological analysis of mortality was carried out in all LTCFs of the Vicenza Province using standard epidemiological measures. This paper would like to be a bottle with a message inside for future research on pandemics. 


\section{Materials and Methods}

\section{Background}

The Vicenza Province is one of the seven Provinces of the Veneto Region situated in the North East of Italy with 867,314 inhabitants. In the local health district (Azienda Unità Socio-Sanitaria Locale 8) there are 34 LTCFs hosting a total 3664 residents. In the literature, the term long term care facilities encompasses a diverse range of healthcare settings including nursing homes, rehabilitation centers, long $\square$ term care hospitals, psychiatric care facilities and facilities for people with intellectual disabilities. In the Vicenza Province, the vast majority of LTCFs are devoted to the care of individuals with either generic or social or intellectual needs. The criteria for admission are based on a composite evaluation obtained by a multidimensional instrument filled out by the GP and a social worker. The score obtained determines the priority for the admission in a public or private LTCF.

The Don Bruzzo LTCF is situated in Gambellara a rural village in the Vicenza province. It is professionally staffed with 5 nurses, 20 nursing aides, 1 social workers, 1 dieticians, 1 physiotherapists, etc. They have nursing staff $\mathrm{H} 24$ per day and a 1 in house doctor on demand and on fixed days is always in attendance. There majority of the 64 residents were elderly and $20(37 \%)$ of them were affected by psychiatric problems or dementia.

The first COVID-19 case in the Don Bruzzo LTCF occurred on March 19. After four days of an unexplained fever and worsening respiratory status, a resident of a LTCF was transferred to a local hospital. Two days after, she resulted positive to COVID-19. All residents of the facility underwent promptly to a COVID-19 swab test and the $82 \%$ resulted positives. Strict infection control measures have been adopted (i.e. isolation of patients, provision of PPE, disinfection, closure to new admission and visitors, educational support, etc.). The news of COVID-19 positivity of such a high numbers of hosts has wacked havoc also because there was a shortage of PPI; 4 out of 5 nurses and even the doctor resulted positives. The personnel was substituted and all interventions have been coordinated by an infectious diseases specialist.

The Don Bruzzo was the first LTCF of the Vicenza Province with a COVID-19 outbreak. Other sporadic cases started to be gradually notified in other LTCF and local health authorities decided to implement a specific registry of mortality in the LTCFs of the Vicenza province.

\section{The field investigation in the Don Bruzzo Long Term Care Facilities}

In the outbreak of Don Bruzzo LTCF, the residents were exposed to COVID-19 in the same few days. An outbreak in which most or all persons are exposed to the same source of infection on the same time (i.e., a so-called universal exposure) permits to consider such population as a cohort and it allows to represent the distribution of COVID-19 clinical manifestations over time. A point in time prevalence indicates the probability that a member of a population has a given condition at a given point in time. A day by day point in time prevalence has been calculated to represent the clinical impact of COVID-19 in an old aged population hosted in the LTCF.

The field investigation of the outbreak was managed according to CDC principles. ${ }^{8}$ The case definition assumed was the positivity to a COVID-19 swab test. The rapid response from the laboratory made not necessary the adoption of criteria to define probable or possible cases. Due to the high sensitivity of the molecular tests, the definition of false positives was also not considered necessary. To exclude false negative cases a second round of swabs was repeated after a week in all negative subjects.

According to the Italian Society of Geriatrics and Gerontology (SIGG), all residents were categorized into three groups: 65-74 years-old; 75-84 years-old; 85-99 years-old and centenaries (above 100 years old). ${ }^{9}$ Comorbidity was obtained from the electronic clinical records. To estimate the effect of comorbidity, residents have been clustered into two groups: those affected by less or 2 chronic conditions and those with more than 2 chronic pathologies.

All residents have been monitored daily for symptoms and checked by measuring temperature, oxygen saturation via pulse oximetry and respiratory rate at bed site. The staging of infection was determined according to WHO criteria. ${ }^{10}$ Asymptomatic were those residents with the absence of signs and symptoms; mild disease was defined by symptoms without evidence of viral pneumonia or hypoxia; moderate disease as the presence of cough, fever with sign of pneumonia; severe disease was stated by the presence of fever, cough, plus respiratory rate of $>30$ breaths $/ \mathrm{min}$ or $\mathrm{SpO}_{2}$ $<90 \%$ on room air.

Recovery was defined by two consecutives negative real-time polymerase chain reaction (RT-PCR) in a nasopharyngeal swab test which were scheduled at the fourth week after positivity. For a better definition of recovery, a blood drawn was done for measuring the antibodies titer at the fourth week and it was repeated also after sixteen weeks.

Disability has been considered as a priori potential risk factor for an adverse clinical outcome. The Barthel scale, is a widespread and validated instrument that was used for evaluation of disability. ${ }^{11}$

The detection of SARS-CoV-2 nucleic acids was performed on Cobas 6800 RTPCR System (Roche Diagnostic GmbH, Mannheim, Germany). A cycle threshold value (Ct-value) $\leq 38$ was defined as a positive test result and a Ct-value of $>40$ or no amplification curve was defined as negative. A median value of 24 of $\mathrm{Ct}$ was assumed to define high or low viral load.

\section{The epidemiological estimation on mortality by COVID-19 in LTCF of the Vicenza Province}

To assess the impact on mortality by COVID-19, a retrospective cohort study has been carried out in all those LTCFs of the Province with at least one COVID-19 case notification. We considered the period time from 2020 January 1 to June 15 . Three basic measures have been used: the attack rate, the case fatality rate and the standardized mortality ratio. The attack rate (AR) corresponds to the percentage of the population that contracts the disease in an at risk population during a specified time interval. The case fatality rate (CFR) is a measure obtained dividing the number of deaths from COVID-19 by the number of cases of infected COVID-19. The excess of mortality in the COVID-19 LTCFs approach relies on the standardized mortality ratios (SMRs). It is defined as the ratio of observed deaths in the study group compared to expected deaths in the study population. To calculate SMRs, we considered first the number of residents from January 1 to June 15 on 2018, 2019 and 2020. Secondly, we calculated and compared the ratio between age- and gender-specific deaths observed with those expected in these periods.

\section{Statistics}

The chi-square test was used to assess differences in demographics and clinical characteristics according to attack rate, CFR and the time to negative conversion of viral RNA. The trend in the age group was evaluated through a test for trend estimated using a logistic regression model. Data on disability have been elaborated in a categorical distribution assuming a Barthel score > 50 either on mobility or dependency. The results of antibody titers have been expressed as the logarithm of each value. The Stata 15.0 statistical package (Stata Corporation, College Station, TX, USA) 
was used to perform all analyses. A $P$ value of $<0.05$ was considered significant.

\section{Results}

\section{The field investigation in the Don Bruzzo Facility}

As of March 19, 2020, 64 residents (51 females and 13 males) were housed in the Don Bruzzo Facility. Forty-three out of the total 53 COVID-19 positive residents were females $(81.2 \%)$ and 10 males $(18.8 \%)$ with a median age of 86 [interquartile range (IQR) 81-92]. The distribution of residents among the age groups was: 9 residents in the $65-74$ years old group $(16.9 \%), 14$ in the 75-84 years old group (26.4\%) and $30 \mathrm{sub-}$ jects in the 85-99 years old group (56.6\%).

Major comorbidities affecting the residents were: hypertension (49\%); cardiovascular diseases (45\%); chronic kidney disease $(41 \%)$; diabetes $(26 \%)$; encephalopathy $(11 \%)$. No statistical differences were found between the age groups on comorbidities and gender.

Figure 1 represents, day by day, the point in time prevalence of the outbreak. It shows the declining number of COVID-19 positives as they recover, the rising number of deceased individuals. For the legibility of the curves, individuals with mild or moderate disease were aggregated. The 11 residents, negative to COVID-19 at the start of the outbreak, remained negative with a proper isolation.

According to the disease staging, 26 (49\%) were asymptomatic, 9 (17\%) had a mild disease; $7(13 \%)$ a moderate stage and $11(21 \%)$ a severe illness severity. A significant difference was found in the illness severity among the age groups. The $89 \%$ of subjects in the 65-75 years-old group have been asymptomatic versus $41 \%$ in the over 75 age groups $(\mathrm{P}<0.01)$.

From March19 to April 20, 11 COVID19 positives residents died. The CFR was $22 \%$. All the deceased residents have been classified at severe stage of illness with respiratory symptoms. One patient with a severe stage of disease survived. The median time lag from symptoms onset and death was 7.5 days (IQR 2.5-13.5) and the median age of deceased residents was 92 years old (range 79-99). The probability of mortality was $0 \%$ in the age group $65-74,7.1 \%$ in the age group $75-85$ group and $30.0 \%$ in the group of 85-99.

Surprisingly, the total number of comorbidities was not found to be an independent risk factors associated to death. A significant difference was instead found between the age groups in the prevalence of chronic renal diseases and associated risk of death $(\mathrm{P}=0.04)$.

Table 1 analyzes the association among the main risk factors as AR, time of recovery in the clearance of viral RNA or death. A positive age gradient was found among the age groups: as age increases, so it raises the susceptibility to contagious $(\mathrm{P}=0.03)$ and grows the probability of death $(\mathrm{P}=0.05)$ and time to negative conversion is prolonged $(\mathrm{P}=0.04)$. Disability was positively associated to susceptibility and contagious $(\mathrm{P}=0.03)$ but not to death $(\mathrm{P}=0.57)$.

No association was found between viral load at baseline, gender and comorbidities. Younger residents had a higher viral load (77.8\% in the $65-75$ years old group had $\mathrm{Ct}<23.5$ vs $43 \%$ in the age group of over 85 years-old). In spite of the higher viral load, the subjects of the youngest group were significantly more asymptomatic than those in the oldest age groups: $88 \%$ in the $65-74$ years old group; $50 \%$ in the $75-84$ years old; $36 \%$ in the over $85(\mathrm{P}=0.2)$.

Twenty-two residents converted to negativity RNA virus within the third and 13 in the fourth week. A remaining group of 8 residents cleared the virus at the sixth week. The latest resident cleared in 50 days. It is also worth to note that 2 residents had an alternate result in nasopharyngeal swab sample as two negative or indeterminate results were followed by a positive one.

\section{The impact of COVID-19 on mortality in the Vicenza Province}

The retrospective epidemiological analysis was conducted in 8 facilities out of 34 of the Vicenza Province, which reported in total 262 COVID-19 cases. From February 20 to June 15 , the number of resi- dents hosted in these facilities was 686 with a median age of 88 years-old (IQR 82-92; $81 \%$ female, $19 \%$ male). Table 2 shows the different impact of the epidemics in these facilities. The attack rate ranged from $7.3 \%$ to $82.8 \%$. Sixty-one out of the 262 residents, tested positives, deceased. The CFR also widely ranged from $7.7 \%$ to $47.8 \%$.

The median age of deceased residents was 90 years-old (IQR 85-94; 80\% female, $20 \%$ male). The SMR demonstrated a total increase of deaths of $60 \%$ if compared with those of 2018 and 2019. In the period considered, 150 deaths were observed toward a number of 93 expected. It should be noted that in the Facility 4, only 1 deceased individual was tested positive against a SMR of $2.12 \%$. This leads to the conclusion that these figures may underestimate the real impact on mortality.

\section{Discussion and Conclusions}

Residents of LTCFs are old and frail, with complex health needs and underlying comorbidities. Diagnosis in LTCFs is often a difficult matter. Hearing and cognition are often impaired in LTCF residents and symptoms may not be expressed or correctly interpreted by caregivers. LTCFs, or other care setting where dependent individuals are institutionalized, have in common to be environments where a relatively large number of people congregate, sometimes in restricted spaces. Consequently residents are prone to infection diseases outbreaks.

The fast spread of respiratory virus outbreaks, including flu, in LTCFs is well recognized. ${ }^{12}$ Besides the COVID-19 pandemic, LTCFs are often and also an endemic

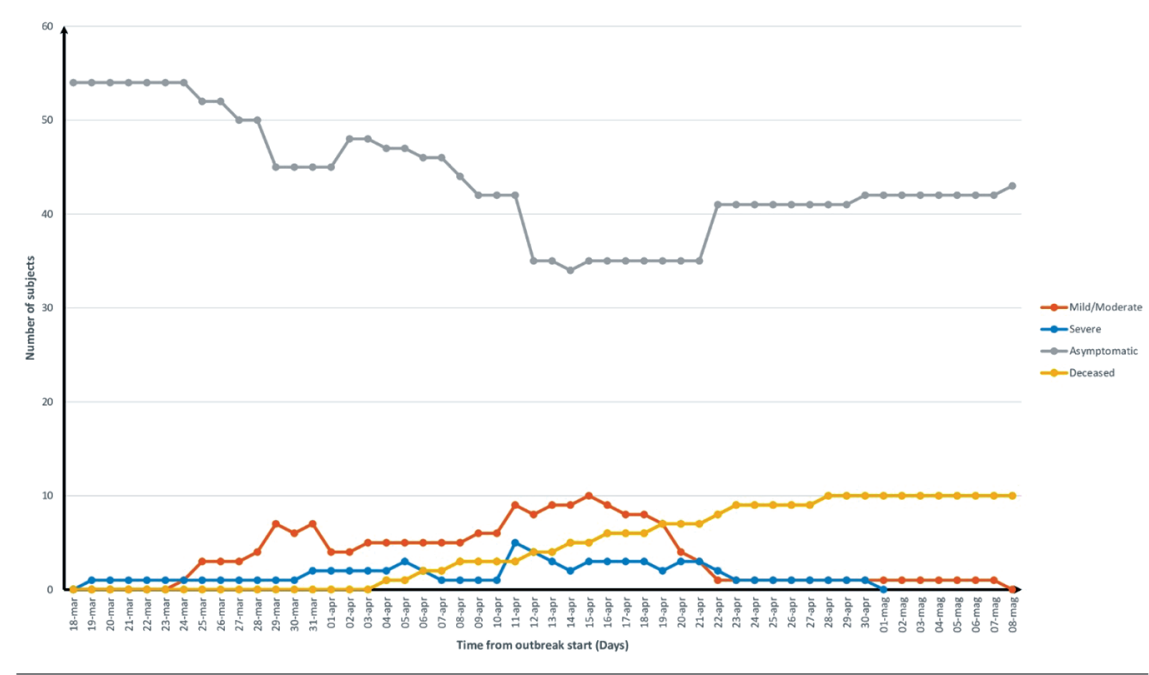

Figure 1. Distribution of clinical manifestation among COVID-19 positive residents. 
reservoir of bacteria resistant to antimicrobials. Patients resident in LTCFs are often extensively colonized with potential pathogens such as Staphylococcus aureus, beta-hemolytic streptococci, members of the Enterobacteriaceae, or Pseudomonas aeruginosa. ${ }^{13}$
Many single reports highlighted the vulnerability of elderly to COVID-19 and it first became evident after the first outbreak in a skilled nursing facility within King County, Washington, late February 2020 and in other settings. ${ }^{14-16}$ The attributable cause of deaths by COVID-19 in LTCFs is one of major limit reported in the literature. Some countries recorded data without a test and with a diagnosis based only to clinical symptoms. The place of death is another possible bias as many deaths were reported in hospital instead of LTCFs. Under-ascertainment and under reporting of COVID-19

Table 1. Main outcomes of epidemiological field investigation.

\begin{tabular}{|c|c|c|c|c|c|c|c|c|c|c|}
\hline \multirow[t]{2}{*}{ Total residents } & \multirow[b]{2}{*}{ No. } & \multicolumn{3}{|c|}{ Study population } & \multirow[b]{2}{*}{ Death } & \multirow{2}{*}{$\begin{array}{l}\text { Death } \\
\% \text { death }\end{array}$} & \multirow{2}{*}{ P-value } & \multicolumn{3}{|c|}{$\begin{array}{c}\text { Time to negative } \\
\text { conversion of viral RNA } \\
\text { (within } 30 \text { days) }\end{array}$} \\
\hline & & COVID+ & $\%$ COVID+ & P-value & & & & $\begin{array}{l}\text { Clearance of } \\
\text { the virus }\end{array}$ & $\%$ death & P-value \\
\hline Total & 64 & 53 & $82.8 \%$ & & 10 & $18.9 \%$ & & 25 & $47.2 \%$ & \\
\hline \multicolumn{11}{|l|}{ Age groups } \\
\hline $65-74$ & 14 & 9 & $64.3 \%$ & $\begin{array}{l}\quad \mathrm{P}=0.03 \\
\text { (test for trend) }\end{array}$ & 0 & $0.0 \%$ & $\begin{array}{l}\mathrm{P}=0.05 \\
\text { (test for trend) }\end{array}$ & 7 & $77.8 \%$ & $\begin{array}{c}\mathrm{P}=0.04 \\
\text { (test for trend) }\end{array}$ \\
\hline $75-84$ & 17 & 14 & $82.4 \%$ & & 1 & $7.1 \%$ & & 7 & $50.0 \%$ & \\
\hline $85-99$ & 33 & 30 & $90.9 \%$ & & 9 & $30.0 \%$ & & 11 & $36.7 \%$ & \\
\hline \multicolumn{11}{|l|}{ Sex } \\
\hline Woman & 51 & 43 & $84.3 \%$ & $\mathrm{P}=0.51$ & 7 & $16.3 \%$ & $\mathrm{P}=0.32$ & 20 & $46.5 \%$ & $\mathrm{P}=0.84$ \\
\hline Men & 13 & 10 & $76.9 \%$ & & 3 & $30.0 \%$ & $\mathrm{C}$ & 5 & $50.0 \%$ & \\
\hline \multicolumn{11}{|c|}{ Disability (Barthel score) } \\
\hline & 36 & 33 & $91.7 \%$ & $\mathrm{P}=0.03$ & 7 & $21.2 \%$ & $\mathrm{P}=0.57$ & 20 & $60.6 \%$ & $\mathrm{P}=0.145$ \\
\hline \multicolumn{11}{|l|}{ Comorbidities } \\
\hline 0-2 diseases & 28 & 21 & $75.0 \%$ & $\mathrm{P}=0.14$ & 3 & $14.3 \%$ & $\mathrm{P}=0.49$ & 10 & $47.6 \%$ & $\mathrm{P}=0.95$ \\
\hline $3+$ diseases & 36 & 32 & $88.9 \%$ & & 7 & $21.9 \%$ & & 15 & $46.9 \%$ & \\
\hline \multicolumn{11}{|c|}{ Viral load at baseline (time zero)* } \\
\hline $\mathrm{Ct}>23.5$ & & 27 & & & 4 & $14.8 \%$ & $\mathrm{P}=0.62$ & 15 & $55.6 \%$ & $\mathrm{P}=0.262$ \\
\hline $\mathrm{Ct}<23.5$ & & 25 & & & 5 & $20.0 \%$ & & 10 & $40.0 \%$ & \\
\hline \multicolumn{11}{|l|}{ Disease severity } \\
\hline Asymptomatic & & 26 & & & 0 & $0.0 \%$ & $\mathrm{P}<0.01$ & 15 & $57.7 \%$ & $P=0.04$ \\
\hline Mild disease & & 9 & 8 & & 0 & $0.0 \%$ & & 5 & $55.6 \%$ & \\
\hline Moderate disease & & 7 & & & 0 & $0.0 \%$ & & 4 & $57.1 \%$ & \\
\hline Severe disease & & 11 & 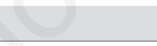 & & 10 & $90.9 \%$ & & 1 & $9.1 \%$ & \\
\hline
\end{tabular}

*, Ct-value: number of cycles of PCR amplifications to define COVID-19 viral load.

Table 2. Results of the study on mortality in the Vicenza Province.

\begin{tabular}{|c|c|c|c|c|c|c|}
\hline Facility & $\begin{array}{c}\text { Total residents } \\
01 / 03 / 2020\end{array}$ & $\begin{array}{l}\text { COVID-2 } \\
\text { positives }\end{array}$ & Deceased & Attack rate & Fatality rate & SMR \\
\hline Don Bruzzo Facility & 64 & 52 & 10 & $82.8 \%$ & $22.4 \%$ & 1.11 \\
\hline Facility 2 & 55 & 4 & 1 & $7.3 \%$ & $25.0 \%$ & 1.12 \\
\hline Facility 3 & 121 & 47 & 11 & $38.8 \%$ & $23.4 \%$ & 1.75 \\
\hline Facility 4 & 59 & 1 & 0 & $1.7 \%$ & $0.0 \%$ & 2.12 \\
\hline Facility 5 & 33 & 13 & 4 & $39.4 \%$ & $30.8 \%$ & 1.01 \\
\hline Facility 6 & 45 & 13 & 1 & $28.9 \%$ & $7.7 \%$ & 0.51 \\
\hline Facility 7 & 78 & 42 & 9 & $53.8 \%$ & $21.4 \%$ & 3.52 \\
\hline Facility 8 & 146 & 23 & 11 & $15.8 \%$ & $47.8 \%$ & 1.57 \\
\hline Facility 9 & 85 & 70 & 13 & $82.4 \%$ & $18.6 \%$ & 2.17 \\
\hline Total & 686 & 262 & 61 & $38.2 \%$ & $23.3 \%$ & 1.6 \\
\hline
\end{tabular}

SMR, standard mortality ratio. 
cases has been a common feature of the pandemic.

International evidence of mortality associated with COVID-19 in LTCFs has been published and periodically updated. The impact of COVID-19 on LTCFs residents has been very different internationally. However, using data with the caveats that definitions used for different settings of care and difficulties in comparing data, it has been estimated that the share of total COVID-19 associated deaths in residents of LTCFs is $47 \%$ (based on 26 countries). ${ }^{16} \mathrm{In}$ Ontario, Canada, the population in LTCFs represented over $80 \%$ of deaths from probable cases of COVID-19. ${ }^{17} \mathrm{~A}$ report based on different sources of data and from different regions of the world estimated a CFR of $14.8 \%$ in the age class over 80 's. ${ }^{18}$

The SMR calculated in the subset of LTCFs of the Vicenza Province is up to $60 \%$. The comparison of CFR and age of death between the group of 8 LTCF and the $\mathrm{CF}$ were similar. Indeed, the median age of deceased residents was 90 years old in the 9 LTCF and 92 years old in the CF, the CFR was $23.3 \%$ while in $\mathrm{CF}$ was equal to $22.4 \%$. We found age gradient in all dimensions considered (i.e., symptoms, illness severity, time of recovery, clearance of the virus, immune response and CFR). A meta-analysis evidenced that hypertension, diabetes, chronic obstructive pulmonary disease, cardiovascular disease and cerebrovascular disease are major risk factors associate with COVID-19 adverse outcome. ${ }^{19}$ We did not find a positive correlation of comorbidity with death.

Our work has many limits. First is the poor statistical power which does not permit to generalize any conclusion of the observational study. Moreover, in the calculation of SMR, we were not able to report on deceased individuals without a COVID19 test. The diagnosis of COVID-19 associated death was made on a clinical basis and it might lead to diagnostic mistakes.

We concluded that COVID-19 outbreak in the Italian LTCF highlighted the problem of infection control in these settings of care and the need for adoption of medical standards, at European level, as suggested by a European specialist panel. ${ }^{20}$

\section{References}

1. RedZone Online. Coronavirus in Italia, 110.574 casi positivi e 13.155 morti. Il bollettino del $1^{\circ}$ Aprile; Corriere della Sera. Available from: https://www.corriere.it/salute/malattie_infettive/20_april e 01/coronavirus-italia-110574-casi-positivi-13155-morti-bollettino-1-aprile-766 df6fc-742c-11ea-b181-d5820c4838fa. shtml

2. Italian Ministry of Health. Epidemia COVID-19; Aggiornamento nazionale (May 2020). Available from: http://www. salute.gov.it/portale/nuovocoronavirus/d ettaglioContenuti

3. Wang L, He W, Yu X, et al. Coronavirus disease 2019 in elderly patients: characteristics and prognostic factors based on 4-week follow-up. J Infect. 2020;80: 639-45.

4. Williamson EJ, Walker AJ, Bhaskaran K, et al. Factors associated with COVID19-related death using OpenSAFELY. Nature 2020;584:430-6.

5. Chen N, Zhou M, Dong X, et al. Epidemiological and clinical characteristics of 99 cases of 2019 novel coronavirus pneumonia in Wuhan, China: a descriptive study. Lancet 2020;395:507-13.

6. Wang B, Li R, Lu Z, Huang Y. Does comorbidity increase the risk of patients with COVID-19: evidence from metaanalysis. Aging (Albany NY). 2020;12: 6049-57.

7. Berloto S, Notarnicola E, Perobelli E, Rotolo A. Italy and the COVID-19 longterm care situation. Last updated: 22 April 2020. Available from: https://tccovid.org/wp-content/uploads/2020/ 04/LTC-COVID19-situation-in-Italy-22April-2020.pdf

8. Center for Diseases Control and Prevention. Principle of epidemiology in public health practice; Lesson 6: Investigating an outbreak. Available from: https://www.cdc.gov/csels/dsepd/ ss 1978/lesson6/section2.html

9. Società Italiana di Geriatria e Gerontologia. Quando si diventa anziani. Available from: https://www.sigg.it/wpcontent/uploads/2018/12/News_Quando -si-diventa-anziani.pdf

10. World Health Organization (WHO). Clinical management of COVID-19: interim guidance, 27 May 2020. Available from: https://apps.who.int/iris/ handle/10665/332196
11. Mahoney FI, Barthel. Functional evaluation: the Barthel index. Md State Med J 1965;14:61-5.

12. Lansbury LE, Brown CD, Nguyen-VanTam JS. Influenza in long-term care facilities. Influenza Other Respir Viruses 2017;11:356-66.

13. Ambretti S, Bassetti M, Clerici P, et al.. Screening for carriage of carbapenemresistant Enterobacteriaceae in settings of high endemicity: a position paper from an Italian working group on CRE infections. Antimicrob Resist Infect Control 2019;13:136.

14. McMichael TM, Clark S, Pogosjans S, et al. COVID-19 in a long-term care facility - King County, Washington, February 27-March 9, 2020. MMWR Morb Mortal Wkly Rep 2020;69:339-42.

15. Arons MM, Hatfield KM, Reddy SC, et al. Presymptomatic SARS-CoV-2 infections and transmission in a skilled nursing facility. New Engl J Med 2020;382: 2081-90

16. Roxby AC, Greninger AL, Hatfield KM, et al. Detection of SARS-CoV-2 among residents and staff members of an independent and assisted living community for older adults - Seattle, Washington, 2020. MMWR Morb Mortal Wkly Rep 2020;69:416-8.

17. Comas-Herrera A, Zalakaín J, Lemmon E, et al. Mortality associated with COVID-19 in care homes: international evidence. Article in LTCcovid.org, International Long-Term Care Policy Network, CPEC-LSE, 2020. Last updated: 1st February 2021. Available from: https://tccovid.org/2020/04/12/mortality-associated-with-covid-19-outbreaksin-care-homes-early-international-evidence/

18. Szklaski C. Canada's proportion of COVID-19 deaths in long-term care double the average of other countries, study shows. The Canadian Press; Posted: Jun 25, 2020. Available from: https://www. cbc.ca/news/health/coronavirus-canadalong-term-care-deaths-study-1.5626751

19. Verity R, Okell LC, Dorigatti I, et al. Estimates of the severity of coronavirus disease 2019: a model-based analysis. Lancet Infect Dis 2020;20:669-77.

20. O’Neill D, Briggs R, Holmerová I, et al.COVID-19 highlights the need for universal adoption of standards of medical care for physicians in nursing homes in Europe Eur Geriatr Med 2020;11:645-50. 